\title{
Adjusting for population stratification and relatedness with sequencing data
}

\author{
Yiwei Zhang ${ }^{*}$, Wei Pan \\ From Genetic Analysis Workshop 18 \\ Stevenson, WA, USA. 13-17 October 2012
}

\begin{abstract}
To avoid inflated type I error and reduced power in genetic association studies, it is necessary to adjust properly for population stratification and known/unknown subject relatedness. It would be interesting to compare the performance of a principal component-based approach with a linear mixed model. Furthermore, with the availability of genome-wide sequencing data, the question of whether it is preferable to use common variants or rare variants for such an adjustment remains largely unknown. In this paper, we use the Genetic Analysis Workshop 18 data to empirically investigate these issues. We consider both a quantitative trait and a binary trait.
\end{abstract}

\section{Background}

In genetic association studies, population stratification and known or cryptic relatedness are always issues. If not suitably accounted for, these may cause inflated type I errors and reduced power. A popular approach to adjusting for population stratification is to construct principal components (PCs) from some similarity matrix for the samples and to include the PCs as covariates in a regression model [1]. This is referred to as a PC-based approach. However, it is thought that these approaches "do not model family structure or cryptic relatedness" [2]. A more general, and perhaps more powerful, approach is to apply linear mixed models (LMMs) to account for both population stratification and relatedness [3,4]. EMMAX software [5] has facilitated the implementation of LMM by using the identityby-state (IBS) matrix to capture the complex correlation structure in the samples. As these methods are studied intensely in genome-wise association studies (GWAS), a natural question is how they will perform with sequencing data. It is also of interest to investigate whether common variants $(\mathrm{CVs})$ with minor allele frequencies (MAFs) no less than 0.05 , or rare variants (RVs) with $0<\mathrm{MAF}<0.01$, should be used to infer the samples' genetic similarities.

In this paper, we compare the PC-based approach with LMM to determine which approach can better

\footnotetext{
* Correspondence: zhan1447@umn.edu

Division of Biostatistics, School of Public Health, University of Minnesota, 420 Delaware Street SE, Minneapolis, MN 55455, USA
}

control the inflation of type I error arising from correlated samples. The association testing is carried out for a quantitative trait and a binary trait in the Genetic Analysis Workshop 18 (GAW18) family-based sequencing data. For a complete comparison, we construct and consider PCs from different similarity matrices: the sample covariance matrix and the IBS matrix. Finally, we discuss the best choice of variants for constructing the similarity matrix, which has been the subject of several recent studies [6-8].

\section{Methods}

In the PC-based approach, for a given similarity matrix, we obtain its $m$ largest eigenvalues $\lambda_{j}$ and the corresponding eigenvectors $v_{j}$ for $j=1, \ldots, m$ and denote $X_{m}=\left(\sqrt{\lambda_{1}} v_{1}, \ldots, \sqrt{\lambda_{m}} v_{m}\right)$. For a quantitative trait, we use a linear regression model $Y=\beta_{0}+X_{m} \gamma+Z \zeta+g \beta+\delta$, where $\delta \sim N\left(0, \sigma^{2} I\right)$. For a binary trait we adopt a logistic model: $\operatorname{Logit}(E(Y))=\beta_{0}+X_{m} \gamma+Z \zeta+g \beta$.

In the 2 models above, $Y=\left(Y_{1}, Y_{2}, \ldots, Y_{n}\right)^{\prime}$ is the vector of the traits for $n$ subjects. $Z=\left(z_{1}, z_{2}, \ldots, z_{n}\right)^{\prime}$ is the matrix of covariates, and $g=\left(g_{1}, g_{2}, \ldots, g_{n}\right)^{\prime}$ is the vector of the genotype scores for 1 or more variants to be tested. We denote the method by which PCs are obtained from the IBS matrix as PCA. IBS, and the method by which PCs are obtained from the covariance matrix as PCA.V. 
In an LMM, $Y=\beta_{0}+Z \zeta+g \beta+u+\delta$, where $Y, Z$, and $g$ are defined as above, $u$ is the random effect for other polygenic effects and $\delta$ is the residual error. It is assumed that $\delta \sim N\left(0, \sigma^{2} I\right)$ and $u=\left(u_{1}, u_{2}, \ldots, u_{n}\right)^{\prime} \sim N\left(0, \sigma_{g}^{2} K\right)$, where $K$ is the IBS matrix. We use EMMAX [5] for parameter estimation and inference.

For hypothesis testing, PCA.V and PCA.IBS adopted the Wald test and EMMAX adopted the F-test.

\section{Results}

We used the GAW18 sequencing data containing 959 individuals and 8,348,674 single nucleotide variants (SNVs) across all 11 chromosomes, among which 2,791,923 SNVs were CVs and 3,977,003 were RVs. After pruning by PLINK [9] using a sliding window of size 50, moving step of 5 and $r^{2}<5 \%$, and filtering out those with missing call $>0.05$, there were $63,157 \mathrm{CVs}$ left. We randomly selected $10,837 \mathrm{CVs}$ from those to construct the similarity matrix. The IBS matrix was obtained by EMMAX, and the covariance matrix was obtained by the $\mathrm{R}$ function $\operatorname{cov}()$ with "use = pairwise.complete.obs" to utilize the maximum number of variants.

We used the measurements of systolic blood pressure (SBP) at time point $1, S B P_{1}$, and the hypertension diagnosis at time point $1, H T N_{1}$. The former is a quantitative trait and the latter is a binary trait. There are 855 samples available. Gender, smoking, and age are the covariates.

\section{Association test with CVs}

We carried out single single-nucleotide polymorphism (SNP) analysis on a set of 6228 CVs randomly selected from all the pruned CVs. Based on the findings of previous GWAS that most of the SNPs were not significantly associated with hypertension, we could assume these 6228 CVs were null SNPs. Because some subjects were from the same families and thus correlated, we expected to observe an inflated type I error if we treated the samples as independent. If the PC-based method or LMM was effective in adjustment, the $p$ values should have followed a uniform distribution. This also meant that the proportion of the tests with $p$ value $<0.05$ should be close to 0.05 and the inflation factor $\lambda$ close to $1 ; \lambda$ is the inflation factor of $p$ values estimated by the function gcontrol 2 in $\mathrm{R}$ package gap. It is calculated as the ratio of the medians of the observed and expected statistics, respectively.

Figure 1 shows that, without adjustment, for $S B P_{1}$ the observed $p$ values deviate from the theoretical uniform distribution $(\lambda=1.14)$. For $H T N_{1}$, the observed $p$ values seem to follow the uniform distribution $(\lambda=0.94)$. This observation might indicate a mild heritability in the GAW18 data set.

Table 1 shows the quantiles of the association mapping $p$ values of $S B P_{1}$ with adjustment. We can see the $p$ values are almost uniformly distributed. The proportion of the $p$ values $<0.05$, estimating the type I error rates, is around 0.05 and of $\lambda$ 's around 1. Figure 2 shows some difference between $p$ values obtained from the 2 PC-based models and EMMAX. There are also differences in the estimated SNP effects, $\beta^{\wedge}$ s. However, both the $p$ values and $\beta^{\wedge}$ s from the 3 methods are highly correlated.

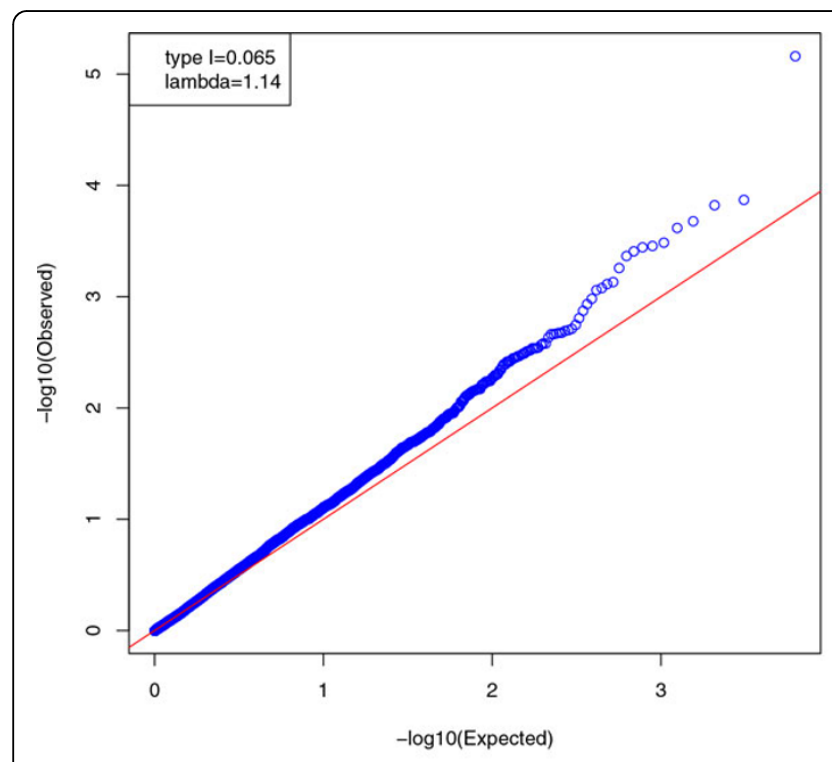

(a)

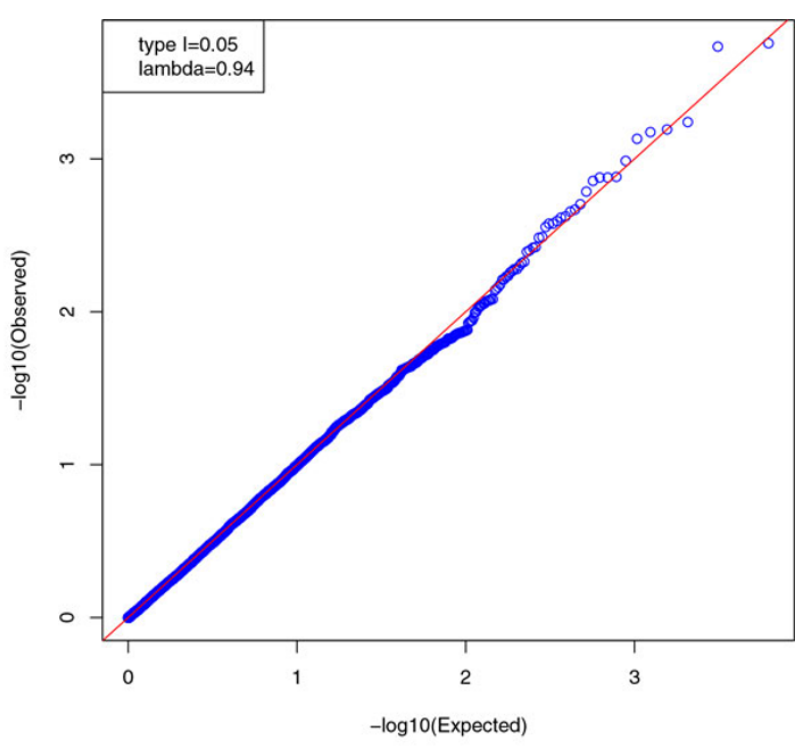

(b)

Figure $1 \mathrm{Q}-\mathrm{Q}$ plots of $p$ values without considering the correlation among samples 
Table 1 Summary statistics of $p$ values for SBP 1 by PCA.V, PCA.IBS, and EMMAX. The similarity matrix is based on CVs.

\begin{tabular}{lcccccccc}
\hline Method & Min. & 1st. Qu. & Median & Mean & 3rd Qu. & Max. & \% $(\boldsymbol{p}$ val $<\mathbf{0 . 0 5})$ & $\boldsymbol{\lambda}$ \\
\hline PCA.V & $1.106 \mathrm{e}-05$ & 0.232 & 0.486 & 0.491 & 0.750 & 1.000 & 0.053 & 1.068 \\
PCA.IBS & $5.022 \mathrm{e}-06$ & 0.235 & 0.491 & 0.493 & 0.749 & 1.000 & 0.054 & 1.041 \\
EMMAX & $1.42 \mathrm{e}-05$ & 0.254 & 0.516 & 0.508 & 0.758 & 1.000 & 0.043 & 0.974 \\
\hline
\end{tabular}

The similarity matrix is based on CVs.

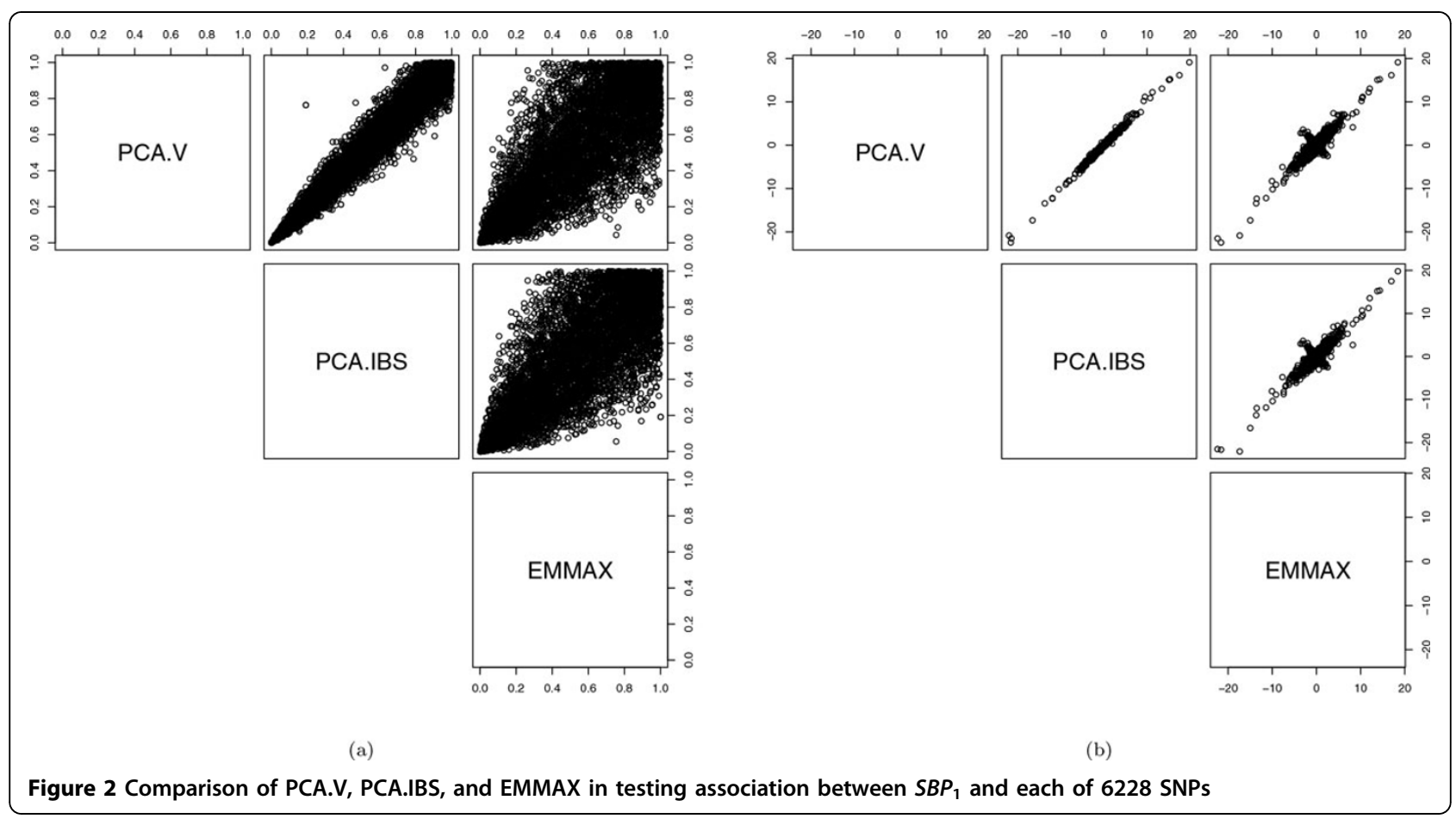

We also apply the methods to the binary trait $H N_{1}$. Table 2 shows the $p$ values follow a uniform distribution after adjustment. Figure 3 shows that the correlations between the $p$ values or $\beta$ ^s from the 3 methods are weaker than those for $S B P 1$. This contrast is partly a result of the logistic link in the PC-based models differing from the identity link in the LMM.

\section{CVs or RVs?}

Lastly, we examine which type of variants, CVs or RVs, are more capable of capturing the underlying sample structure. For this purpose, we use PLINK to randomly select 11,103 variants from 1,104,098 pruned RVs to construct the covariance matrix or IBS matrix.
Table 3 shows the results of the association testing, adjusted with PCs of the new similarity matrices based on RVs. PCA.IBS does a satisfactory job of controlling type I errors and $\lambda \mathrm{s}$ in testing $6228 \mathrm{CVs}$ for both $S B P_{1}$ and $H T N_{1}$. EMMAX is a little conservative for $H T N_{1}$. Interestingly, we can see a greater distinction between PCA.V and PCA.IBS here than in the previous results, where the similarity matrices were based on CVs. The PCA.IBS is better than the PCA.V at controlling the inflation.

Originally, the weaker performance of PCA.V based on RVs was thought to be a result of insufficient inclusion of PCs. Following the suggestion of Patterson et al [1], we use the Tracy-Widom test to test how many PCs

Table 2 Summary statistics of $p$ values for HTN 1 by PCA.V, PCA.IBS, and EMMAX

\begin{tabular}{lcccccccc}
\hline Method & Min. & 1st. Qu. & Median & Mean & 3rd Qu. & Max. & \% $(\boldsymbol{p}$ val $<0.05)$ & $\boldsymbol{\lambda}$ \\
\hline PCA.V & $1.457 \mathrm{e}-04$ & 0.239 & 0.489 & 0.494 & 0.748 & 1.000 & 0.055 & 1.054 \\
PCA.IBS & 7.044e-05 & 0.239 & 0.492 & 0.493 & 0.746 & 1.000 & 0.056 & 1.039 \\
EMMAX & 2.831e-04 & 0.259 & 0.510 & 0.507 & 0.761 & 1.000 & 0.048 & 0.977 \\
\hline
\end{tabular}

The similarity matrix is based on CVs. 


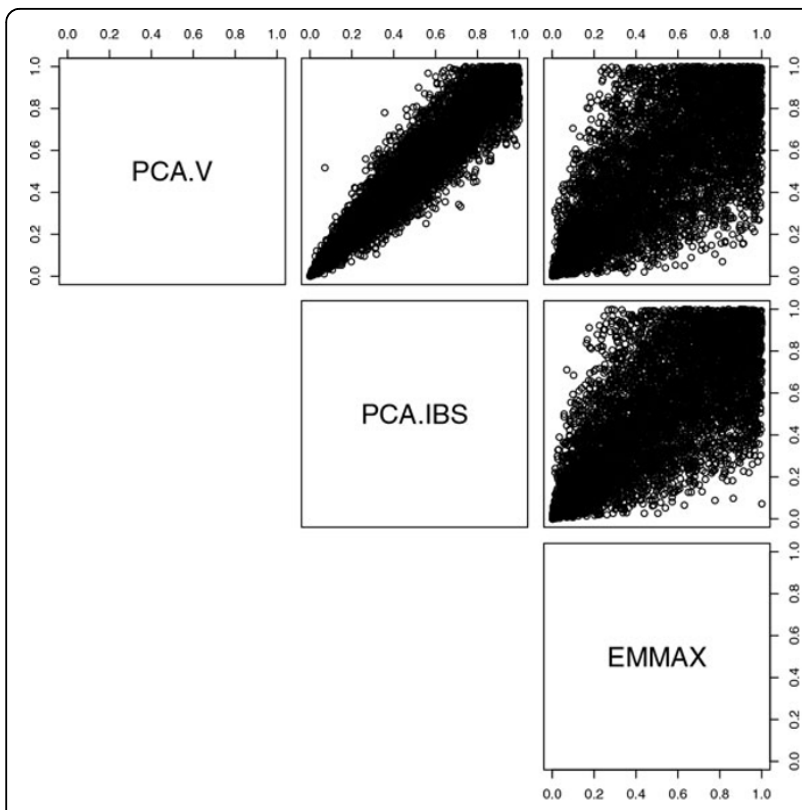

(a)

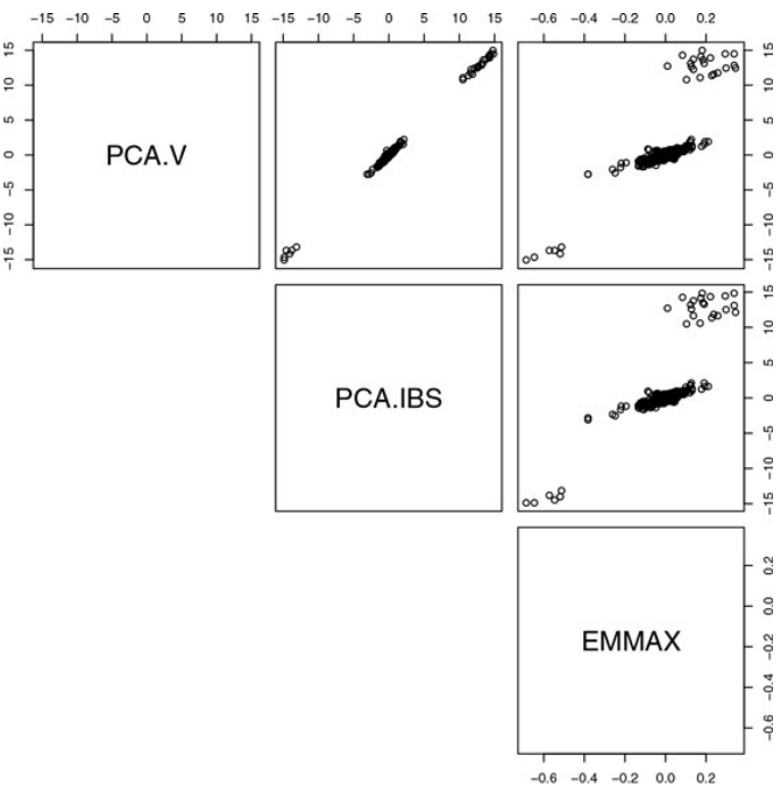

(b)

Figure 3 Comparison of PCA.V, PCA.IBS, and EMMAX in testing association between $H_{T N}$ and each of 6228 SNPs

are necessary to be considered significant $[2,8]$. The test shows that the top 210 PCs of the covariance matrix all have $p$ values smaller than 0.05 . However, we fail to obtain reasonable $p$ values with 200 PCs included. This might be because the model could not be fitted, given the small sample size.

Alternatively, we turn to the scree plots (Figure 4) to explain the disparity between the use of CVs and of RVs for a similarity matrix. For the covariance matrix calculated with CVs, there are 458 eigenvalues $>1$, with the top 25 PCs explaining $19.06 \%$ of the total variance; for the IBS matrix, there are only 32 eigenvalues $>1$, and the top 25 PCs explain $11.91 \%$ of the total variance. For the covariance matrix calculated with RVs, there are 433 eigenvalues $>1$ with the top 25 PCs explaining $7.73 \%$ of the total variance; for the IBS matrix, there is only 1 eigenvalue $>1$, with the top 25 PCs explaining $27.02 \%$ of the total variance. In short, when using CVs for constructing the similarity matrix, the top 25 PCs of either type can approximate the correlation structure equally well. Although the top 25 PCs of the IBS matrix can still preserve a large proportion of the variation, when using RVs for the similarity matrix, the counterpart of the covariance matrix does a poorer job of approximation.

\section{Conclusions}

In this paper, we address 3 questions: (a) how the PCbased approach and LMM perform in controlling type I error for correlated samples; (b) whether the IBS or covariance matrix should be used to generate PCs; and (c) whether CVs or RVs should be used to construct the similarity matrix. Based on the association testing of 6228 almost uncorrelated CVs from the GAW18 data, we find that PC-based models were capable of taking into account the sample correlations and worked as well as the LMM. This result is different from the claim made in Price et al [2] that PC-based models do not model family structure or cryptic relatedness. When using CVs to construct the similarity matrix, the top few PCs from the IBS matrix and the covariance matrix yield similar results. But when using RVs, the top few PCs from the IBS matrix are slightly better than those

Table 3 Results of the association tests by PCA

\begin{tabular}{|c|c|c|c|c|c|c|}
\hline & \multicolumn{3}{|c|}{$\%(p$ val $<0.05)$} & \multicolumn{3}{|c|}{$\lambda$} \\
\hline & PCA.V & PCA.IBS & EMMAX & PCA.V & PCA.IBS & EMMAX \\
\hline$\overline{S B P_{1}}$ & 0.068 & 0.052 & 0.052 & 1.121 & 1.050 & 1.054 \\
\hline$H T N_{1}$ & 0.062 & 0.049 & 0.049 & 1.080 & 1.000 & 0.980 \\
\hline
\end{tabular}

The similarity matrix is based on RVs. 


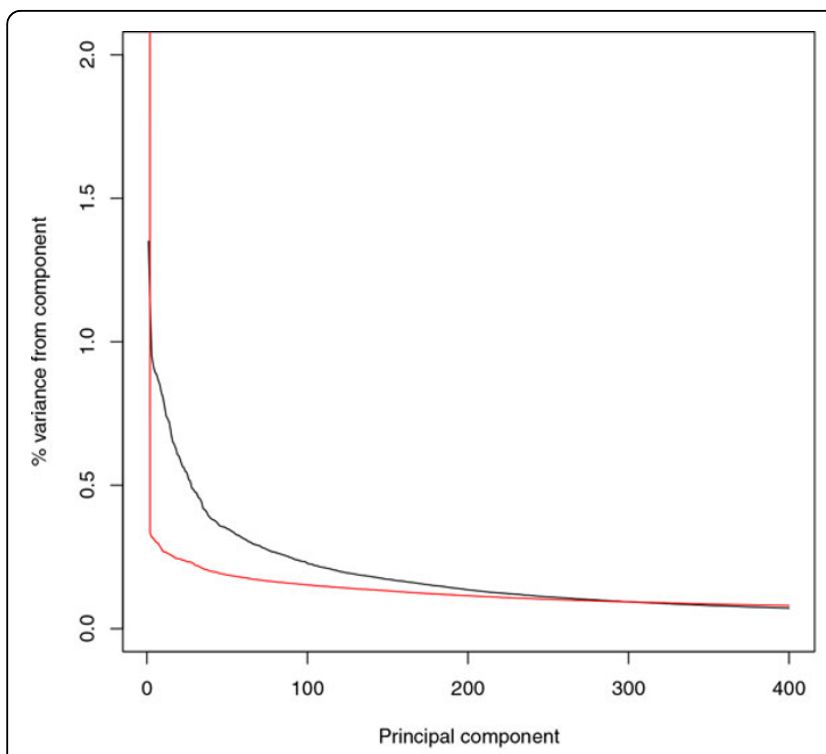

(a)

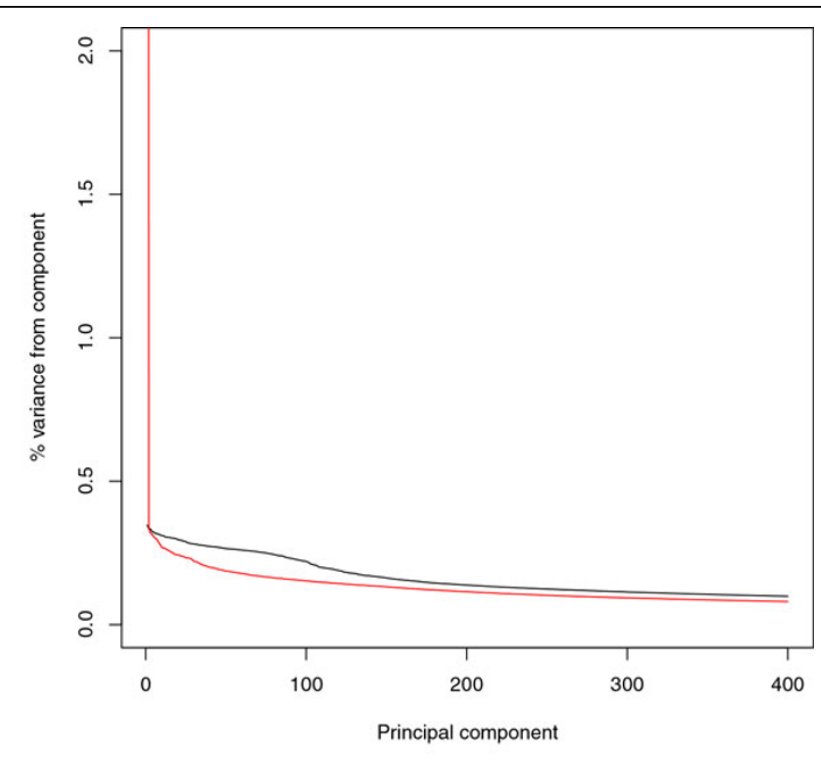

(b)

Figure 4 Scree plots for the top 400 PCs of a similarity matrix based on (a) CVs or (b) RVs. The black line is for the covariance matrix and the red (gray) line is for the IBS matrix.

from the covariance matrix. LMM implemented by EMMAX is generally as effective as anticipated, although sometimes it can be conservative.

One limitation in our study was that in GAW18 data, there is no serious inflation in type I error for testing $H T N_{1}$, even without any adjustment. Although our studies show a positive answer, more studies might be needed to confirm the effectiveness of a PC-based model for testing the binary trait.

\section{Competing interests}

The authors declare that they have no competing interests.

\section{Authors' contributions}

Wei Pan proposed the analysis plan, and Yiwei Zhang provided all statistical analysis and drfted the manuscript. All authors read and approved the final manuscript.

\section{Acknowledgements}

We thank the GAW18 workshop for offering us such a complete data set to practice our methods. YZ and WP were supported by National Institutes of Health grants R21DK089351, R01HL65462, R01HL105397, and R01GM081535. The GAW18 whole genome sequence data were provided by the T2DGENES Consortium, which is supported by NIH grants U01 DK085524, U01 DK085584, U01 DK085501, U01 DK085526, and U01 DK085545. The other genetic and phenotypic data for GAW18 were provided by the San Antonio Family Heart Study and San Antonio Family Diabetes/Gallbladder Study, which are supported by NIH grants P01 HL045222, R01 DK047482, and R01 DK053889. The Genetic Analysis Workshop is supported by NIH grant R01 GM031575.

This article has been published as part of BMC Proceedings Volume 8 Supplement 1, 2014: Genetic Analysis Workshop 18. The full contents of the supplement are available online at http://www.biomedcentral.com/bmcproc/ supplements/8/S1. Publication charges for this supplement were funded by the Texas Biomedical Research Institute.
Published: 17 June 2014

\section{References}

1. Patterson N, Price AL, Reich D: Population structure and eigenanalysis. PLoS Genet 2006, 2:e190.

2. Price AL, Zaitlen NA, Reich D, Patterson N: New approaches to population stratification in genome-wide association studies. Nat Rev Genet 2010, 11:459-463.

3. Yu J, Pressoir G, Briggs WH, Bi IV, Yamasaki M, Doebley JF, McMullen MD, Gaut BS, Nielsen DM, Holland JB: A unified mixed-model method for association mapping that accounts for multiple levels of relatedness. Nat Genet 2005, 38:203-208.

4. Zhou X, Stephens M: Genome-wide efficient mixed-model analysis for association studies. Nat Genet 2012, 44:821-824.

5. Kang HM, Sui JH, Zaitlen NA, Kong S, Freimer NB, Sabatti C, Eskin E: Variance component model to account for sample structure in genomewide association studies. Nat Genet 2010, 42:348-354.

6. Siu H, Lin L, Xiong M: Manifold learning for human population structure studies. PLoS One 2012, 7:E29901.

7. Baye TM, He H, Ding L, Kurowski BG, Zhang X, Martin LJ: Population structure analysis using rare and common functional variants. BMC Proc 2011, 5(Suppl 9):S8.

8. Zhang Y, Guan W, Pan W: Adjustment for population stratification via principal components in association analysis of rare variants. Genet Epidemiol 2013, 37:99-109.

9. Purcell $S$, Neale B, Todd-Brown K, Thomas L, Ferreira MAR, Bender D, Maller J, Sklar P, De Bakker PIW, Daly MJ: Plink: a tool set for wholegenome association and population-based linkage analyses. Am J Hum Genet 2007, 81:559-575.

doi:10.1186/1753-6561-8-S1-S42

Cite this article as: Zhang and Pan: Adjusting for population stratification and relatedness with sequencing data. BMC Proceedings 2014 8(Suppl 1):S42. 